\title{
Age and Sex Differences in Verbal and Visuospatial Abilities
}

\author{
Efrat Barel and Orna Tzischinsky
}

Department of Behavioral Sciences, The Max Stern Academic College of Emek Yezreel, Emek Yezreel, Israel

ABSTRACT

In order to explore the developmental patterns of sex differences in verbal and visuospatial abilities, the present study investigated sex differences in various cognitive abilities among children and adults. Three hundred and twenty-six children and adults completed a battery of six cognitive tasks testing two sets of abilities: The verbal cognitive battery tested verbal fluency and short-term memory tasks. The visuospatial battery tested mental rotation, localization, and form-completion tasks. Results showed a significant Sex $\times$ Age interaction on the mental rotation task, with men outperforming women in the 3D task, but with no sex differences shown in childhood in the 2D task. Sex differences in verbal fluency were found, with girls and women outperforming boys and men in this task. Findings are discussed within an integrative approach of biological as well as environmental factors.

\section{INTRODUCTION}

Are sex differences in cognitive abilities evident early in life and magnified throughout development? Or are they established in adult life? And do different cognitive abilities follow the same developmental path? The present study aimed at investigating sex differences in various verbal and visuospatial abilities in childhood and adulthood. Sex differences in cognition received consistent support in adults. Men outperform women in visuospatial abilities, whereas women outperform men in verbal abilities (Halpern, 2012; Hines, 2004). Although research focusing on visuospatial abilities found that men outperform women on many tasks (e.g., navigation strategies and geographic orientation; Driscoll, Hamilton, Yeo, Brooks, \& Sutherland, 2005; Iachini, Sergi, Ruggiero, \& Gnisci, 2005; Parsons et al., 2004), the largest effect size has been found on mental rotation (e.g., Barel \& Tzischinsky, 2017; Burton \& Henninger, 2013; Hines et al., 2003; Peters, Manning, \& Reimers, 2007; also see Linn \& Petersen, 1985; and Voyer, Voyer, \& Bryden, 1995, for meta-analyses). Linn and Petersen's (1985) metaanalysis provided evidence for sex differences in different aspects of spatial cognition with mental rotation tasks, which involve rotating figures in depth or in the picture plane, showing large sex differences (Cohen's $d=0.73$ ), followed by spatial perception tasks, which involve determining spatial relations in the presence of distractors (e.g., the
Water Level Test), showing medium size sex differences (Cohen's $d=$ 0.44 ) favoring male participants. Women have been found to outperform men in verbal abilities, especially verbal memory (e.g., Bleecker, Bolla-Wilson, Agnew, \& Meyers, 1988; Kramer, Delis, \& Daniel, 1988) and verbal fluency (e.g., Burton \& Henninger, 2013; Weiss, Kemmler, Deisenhammer, Fleischhacker, \& Delazer, 2003). In other areas, such as vocabulary, verbal reasoning, and line orientation (Kimura, 2002), the findings are inconsistent.

In comparison with the consistency found in adults for sex differences in cognitive abilities, the age at which these differences emerge is unclear. Empirical evidence for sex differences in cognitive abilities prior to puberty did not produce unequivocal findings. Some studies have suggested that sex differences in verbal abilities appear early in life. For example, Lutchmaya, Baron-Cohen, and Raggatt (2002) demonstrated that girls show superiority to boys in vocabulary development, with 2-year-old girls using significantly more words than boys.

Corresponding author: Efrat Barel, Department of Behavioral Sciences, The Max Stern Academic College of Emek Yezreel, Emek Yezreel, Israel. Phone number 972-50-2481549; Fax number 972-4-6765784.

E-mail: efratb@yvc.ac.il 
Zambrana, Ystrom, and Pons (2012) documented sex differences in language comprehension at 18 and 36 months of age favoring girls at both time points. The developmental patterns of sex differences were also examined in a meta-analysis conducted by Hyde and Linn (1988). They reviewed the literature concerning sex differences in various verbal abilities (e.g., vocabulary, comprehension) in different age groups. They found that, across verbal tasks, sex differences were shown in children younger than 5 and in adults over the age of 26 . In between, there were no notable sex differences.

Studies exploring individual differences and developmental aspects in visuospatial abilities used various tasks, including mental rotation, the Water Level Test, and block design. Studies of prepuberty children provided mixed results with regard to sex differences in visuospatial abilities. Some studies have demonstrated that the male advantage in mental rotation is apparent as early as infancy. For example, two studies have shown male advantages in $2 \mathrm{D}$ and $3 \mathrm{D}$ object rotation in infants aged 4 and 5 months (Moore \& Johnson, 2008; Quinn \& Liben, 2008). Through a habituation paradigm, infants were familiarized with an object; thereafter, they were presented with its mirror image and with the same object rotated in angle (2D) or in depth (3D). Boys looked longer at the mirror image than the familiar image. Nevertheless, not all infant studies provided support for sex differences in mental rotation task in infancy (e.g., Hespos \& Rochat, 1997; Möhring \& Frick, 2013). In a similar vein, studies of preschool and school-aged children also provided mixed results. For example, Frick, Ferrrara, and Newcombe (2013) found sex differences in a mental rotation task involving matching between puzzle pieces and their placement hole at age 5 but not at age 4. Furthermore, Neuburger, Jansen, Heil, and Quaiser-Pohl (2011) documented sex differences in mental rotation tasks involving various stimulus types rotated in a picture with their mirror images in the fourth but not in the second grade. Palejwala and Goldenring Fine (2015) aimed to capture a wide developmental span, ages 2 to 7 years, in order to examine sex differences across these ages. They have examined, among others, sex differences in visual processing via block design and object assembly tasks, and found that sex differences at ages 2 to 3 were absent, whereas they emerged at ages 4 to 7 .

Sex differences in short-term memory in children usually do not generate significant results. For example, no sex differences were found in a picture memory task and in a location memory task in children aged 2 to 7 (Palejwala \& Goldenring Fine, 2015). Another study used several working memory and short-term memory tasks, including listening recall, digit recall, and word recall tasks, in children aged 4.5 to 12 (Alloway, Gathercole, \& Pickering, 2006). These diverse measures did not produce significant results. In contrast, Keith, Reynolds, Roberts, Winter, and Austin (2011) reported sex differences in shortterm memory with the use of a latent variable approach. Girls outperformed boys at ages 5 to 13, whereas boys outperformed girls at ages 14 to 17 .

The accumulated evidence regarding sex differences in cognitive abilities throughout the life span suggests that sex differences in cognition are magnified or become more common during adolescence (Herlitz, Reuterskiöld, Lovén, Thilers, \& Rehnman, 2013). The un- derlying mechanism for the developmental pattern of sex differences in cognitive abilities involves a complex interplay between biological and environmental variables. Among the environmental variables, play experiences have been shown to be associated with higher performance in related cognitive skills. For example, boys tend to prefer playing with construction toys that involve object manipulation and transformation, which has been associated with better performance on spatial visualization tasks (e.g., Caldera et al., 1999). Another environmental variable relates to the socio-cultural gender stereotypes. For example, findings show that in Western cultures, boys are encouraged to play with masculine-stereotyped toys (e.g., trucks, wooden blocks) whereas girls are encouraged to play with feminine-stereotyped toys (e.g., dolls, kitchen sets; Caldera, Huston, \& O'Brien, 1989). As they develop, these gender stereotypes shape men's and women's perceptions of separate cognitive domains, which further influence men's and women's test performances (Quaiser-Pohl \& Lehmann, 2002).

Among the biological variables, endocrine factors such as sex hormones have been suggested as important factors (Halpern, 2012). Sex hormones, including androgens, estrogens, and progestins, can affect a wide range of organs, including the brain. Their greatest effect occurs during two sensitive periods in development: The first is during the prenatal and/or neonatal period, the second during the postnatal period (Collaer, Reimers, \& Manning, 2007; Halpern, 2012). It has been suggested that these sensitive periods in hormonal secretion are associated with sex differences in cognitive abilities (Halari et al., 2005; Hines, 2011; Kimura, 2002). Aside from the notion that sex hormones activate neural circuits sexually-differentiated prenatally, puberty has been indicated as another sensitive period of sex hormone-dependent brain organization, with increased levels of sex hormones secretion influencing changes in cognitive performance (Berenbaum \& Beltz, 2011; Shangguan \& Shi, 2009). This assumption regarding magnified sex differences in cognitive abilities during puberty received support from studies on brain development in adolescence. For example, trajectories of white and gray matter development during adolescence are sexually dimorphic (Lenroot et al., 2007). Furthermore, sexual dimorphism appears in brain regions containing significant populations of sex hormones (Bramen et al., 2011), which, in turn, are involved in individual differences in functions such as cognitive abilities (Herlitz et al., 2013). The evolutionary framework posits that differing evolutionary forces are imposed on human males and females, resulting in differences in anatomy followed by sexual differences in function (Geary, 2010; Lenroot \& Giedd, 2010). Thus, in order to adapt to evolutionary pressures, a differential corresponding neuroendocrine infrastructure needs to develop in human males and females.

Activational influences of sex hormones on cognitive performance received support from various lines of research in humans, including hormone administration (e.g., Cherrier et al., 2001), variation in cognitive abilities throughout the menstrual cycle (e.g., Hausmann, Slabbekoorn, Van Goozen, Cohen-Kettenis, \& Güntürkün, 2000), and menopause studies (e.g., Carlson \& Sherwin, 2000). However, the question regarding the emergence of sex differences in cognition is still unresolved. Previous suggestions postulated the assumption of differ- 
entiated influence on specific cognitive abilities. On the basis of this assumption, sex differences in cognitive abilities are present prior to puberty, but some sex differences appear to be larger in adults than in children (Hines, 2004). Specifically, it has been proposed that sex differences in some visuospatial abilities (e.g., mental rotation) are larger in adults (Voyer et al., 1995).

In sum, previous studies have suggested that sex differences in cognitive abilities are magnified and established during adolescence and adulthood, and are proposed to be mediated by neuroendocrine development in adolescence. In light of the suggested evolutionary mechanism regarding differential evolutionary forces inflicted upon men and women, and the supporting biological infrastructure developing during adolescence, it is assumed that sex differences in cognitive abilities will emerge during adolescence especially in visuospatial tasks. Therefore, the present study was designed to investigate developmental patterns in sex differences in cognitive abilities. Specifically, the present study assessed sex differences in visuospatial and verbal abilities using a battery of six cognitive tasks adapted for children and adults. The verbal cognitive battery includes verbal fluency and short-term memory tasks. The visuospatial battery includes mental rotation, localization, and form completion tasks (Gordon, 1986).

On the basis of the theoretical as well as the empirical literature, the hypotheses are as follows:

H1: It is hypothesized that a Sex $\times$ Age interaction on visuospatial abilities is found in adults, with men outperforming women, and no sex differences in children.

$\mathrm{H} 2$ : It is hypothesized that sex differences in verbal abilities are found with female participants outperforming males, in both children and adults, with a magnified effect in adults.

\section{METHOD}

\section{Participants}

Three hundred and twenty-six children and adults participated in the present study. One hundred and fifty-seven were undergraduate students from various departments: behavioral sciences (Psychology, Education), social sciences (Sociology and Anthropology, Information Systems, Economics, Accounting, and Management), and communication, at a college in the north of Israel. Eighty of the participants were female $\left(M_{\text {age }}=26.04 \pm 3.45\right)$ and seventy-seven were male $\left(M_{\text {age }}=26.18\right.$ \pm 2.78 ). One hundred and sixty-nine were children in grades 4 and 5 from four schools in the north of Israel (two schools from a mediumhigh socioeconomic status background, and two schools from a low socioeconomic status background). Eighty-seven of the participants were female $\left(M_{\text {age }}=10.34 \pm 0.61\right)$ and eighty-two were male $\left(M_{\text {age }}=\right.$ $10.45 \pm 0.61$; see Figure 1). All participants were right-handed according to their subjective reports.

Adult participants were recruited through advertisements at the college, and did not receive monetary compensation for their participation. Participants gave their informed consent. Children were recruited

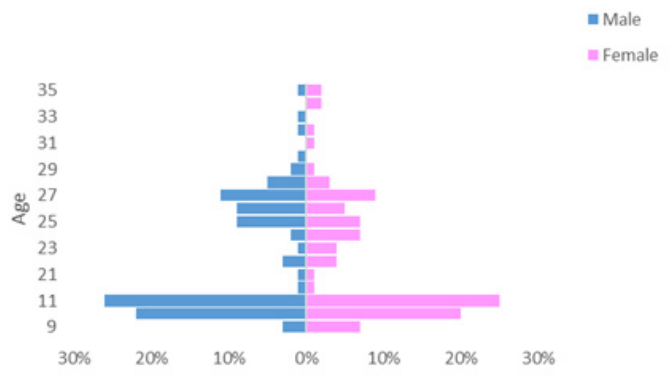

FIGURE 1.

Age $\times$ Sex pyramid diagram

as part of their school assignment, following their parents providing their consent for their child's participation.

\section{Measures}

The study included demographic questions (sex, age) and six cognitive tests that participants performed on a computer and using paper and pencil.

\section{COGNITIVE TEST BATTERY}

Six types of tasks were presented to the participants: three verbal and three visuospatial cognitive tasks (Gordon \& Harness, 1977).

Verbal cognitive tasks.

- Serial sounds: Eight easily recognizable sounds (telephone ringing, chicken clucking, birdsong, guitar, trumpet, baby crying, bell, boat horn) were presented in sequences of two, three, four, five, six, seven, and eight items. Immediately after each sequence, participants were instructed to list the sounds in the same order (Gordon, 1986).

- Serial digits: Digits (between 0 and 9) were presented in sequences of two, three, four, five, six, seven, eight, and nine digits. Immediately after each sequence of numbers, participants were instructed to list the digits in the same order (Gordon, 1986).

- Verbal fluency: Participants were asked to generate as many words as possible beginning with three specified letters of the Hebrew alphabet (A, B, D). Proper names and different forms of the same word (e.g., plurals) were not allowed. One minute was allocated for each letter. The score represented the number of words generated for all three letters (Gordon, 1986).

Visuospatial cognitive tasks.

- Mental rotation task: A computerized task involved three models that were presented randomly on the screen. Pairs of photographs of each model were prepared, in which the models appeared to be nearly identical, except that they were rotated in space with respect to each other. Participants were presented three models at a time and were instructed to decide which two models were the same by mentally rotating them. Each trial was displayed for $30 \mathrm{~s}$ and was separated from the next pair by a rest period of $5 \mathrm{~s}$, during which a white screen was displayed. Eighteen trials were used; on each trial a score of 1 or 0 was given, and then summed for each participant. Children were presented with a 2D stimulus (Gordon, 1986; see Figure 2), and adults with a 3D stimulus (Shepard \& Metzler, 1971; see Figure 3). The choice of 


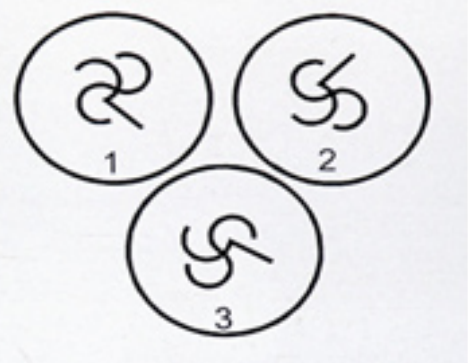

FIGURE 2.

2D mental rotation test (Gordon, 1986). Item example.

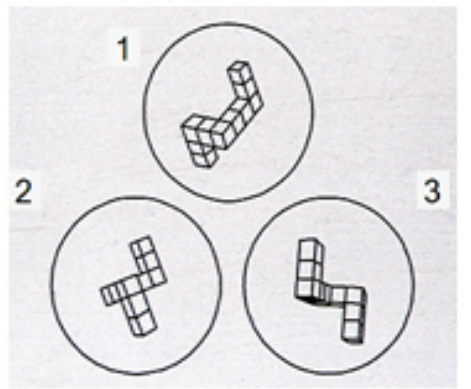

FIGURE 3.

3D mental rotation test (Shepard \& Metzler, 1971). Item example.

two different tasks for children and adults resulted from previous evidence that the 3D tasks may be too cognitively demanding for children (Hoyek, Collet, Fargier, \& Guillot, 2012; Jansen, Schmelter, QuaiserPohl, Neuburger, \& Heil, 2013). Therefore, in the present study, in order to use age-appropriate and-comparable paradigms, a 3D cubes task was used in adults and a 2D abstract characters task (which has been proven at the highest difficulty level among 2D stimuli; Hoyek et al., 2012) were used in children.

- Localization: An $x$ was shown at a certain location on a blank rectangle for $3 \mathrm{~s}$. Participants were instructed to place the mouse arrow in the same location on a parallel blank rectangle with maximum accuracy. Absolute distance was calculated with a higher score representing a poorer performance. Eighteen trials were presented; each trial lasted 20 s (Gordon, 1986).

- Form completion (closure speed): Twelve incomplete silhouettes of familiar objects or scenes were presented in the form of black-andwhite paper cutouts on the computer screen. The items were chosen to be as culture-free as possible. Participants were instructed to imagine the complete silhouette and to identify and describe the object in one or two words. Participants were given $20 \mathrm{~s}$ for each silhouette; on each trial a score of 1 or 0 was given (Gordon, 1986).

\section{Procedure}

The study was approved by the Institutional Review Board. Participants completed a brief demographic questionnaire followed by the cognitive tests. For the computerized tasks, participants were asked to position their heads approximately $50 \mathrm{~cm}$ from the center of the monitor and to focus their gaze on the center of the screen. The cognitive tests took approximately $60 \mathrm{~min}$.

\section{Statistical analysis}

Cognitive ability variables were standardized to z-scores within age group in order to provide a unified presentation scale. First, a series of one-way analyses of variance (ANOVAs) and $t$ tests was conducted to rule out interference with study findings from the participants' field of study in the adults sample and socioeconomic status (based on the participant's school) in the children sample. In the adults sample, six one-way ANOVAs were conducted with the participant's field of study and the cognitive task as independent variables. In the children sample, six $t$ tests were conducted with the participant's school and the cognitive task as independent variables. All analyses yielded nonsignificant results ( $p>.05$ ). Therefore, participants' field of study and participants' socioeconomic status were excluded from further analyses. Next, we examined a previous suggestion, viewing the verbal tasks cluster and the visuospatial tasks cluster as two axes of capabilities (e.g., Gordon \& Lee, 1993). We conducted internal reliability tests in each cluster, but the values were not satisfying (Cronbach's $\alpha=.43$ for verbal abilities, and .53 for visuospatial abilities). These results support the notion that spatial and verbal cognition are not a singular function; rather, they encompass a range of abilities (Iachini et al., 2005). Following this notion, we conducted two separate two-way multivariate analyses of variance (MANOVAs) for the visuospatial tests and for the verbal tests. We have decomposed significant interactions with separate $t$ tests for each age group.

\section{RESULTS}

\section{Correlations Between Cognitive Tasks}

Table 1 shows the correlations between cognitive tasks for the full sample.

\section{TABLE 1.}

Correlations

\begin{tabular}{ccccc}
\hline Serial & Serial & Verbal & Mental \\
sounds & digits & fluency & rotation & Localization \\
\hline
\end{tabular}

Serial sounds

$\begin{array}{lrrrrr}\text { Serial digits } & { }^{* *} . \mathbf{3 0} & & & \\ \text { Verbal fluency } & { }^{* *} .29 & .01 & & & \\ \text { Mental rotation } & { }^{* *} .19 & -.07 & -.01 & & \\ \text { Localization } & { }^{* *} .12 & -.02 & { }^{*} .14 & .08 & \\ \text { Form completion } & .04 & * * *-.33 & { }^{* *} .18 & * * * .35 & * * * .40\end{array}$

Note. In bold: Correlations that remained significant after performing Bonferroni corrections for multiple comparisons; the localization score is higher for poorer performance. ${ }^{*} p<.05^{* *} p<.01{ }^{* * *} p<.001$ 


\section{Verbal Abilities}

A two-way MANOVA was conducted, with sex (male, female) and age group (child, adult) as independent variables on the verbal tasks of serial sounds, serial digits, and verbal fluency. A significant multivariate effect of sex on the three dependent variables was found, Wilks' lambda $=.92, F(3,320)=9.38, p<.001, \eta_{p}{ }^{2}=.08$. Each dependent variable was subjected to a further ANOVA in order to examine whether the trend is the same for each of the verbal tasks. For verbal fluency, the difference between males and females was significant, $F(1,322)=26.27, p<$ $.001, \eta_{\mathrm{p}}^{2}=.08$, with female participants outperforming males (see Table 2). However, differences in serial sounds and serial digits tasks were not statistically significant, $F(1,322)=2.06, p>.05, \eta_{\mathrm{p}}{ }^{2}=.01$, for serial sounds and $F(1,322)=1.32, p>.05, \eta_{\mathrm{p}}{ }^{2}=.01$, for serial digits. There was no multivariate effect of age group on the three dependent variables, Wilks' lambda $=.99, F(3,320)=0.07, p>.05, \eta_{\mathrm{p}}^{2}=.00$. Furthermore, the interaction between sex and age group was not significant, Wilks' lambda $=.98, F(3,320)=0.35, p>.05, \eta_{\mathrm{p}}^{2}=.00$.

\section{Visuospatial Abilities}

A two-way MANOVA was conducted, with sex (male, female) and age group (child, adult) as independent variables on the visuospatial tasks of mental rotation, localization, and form completion. A significant multivariate effect of sex on the three dependent variables was found, Wilks' lambda $=.93, F(3,311)=7.31, p<.001, \eta_{\mathrm{p}}{ }^{2}=.07$. Each dependent variable was subjected to a further ANOVA in order to examine whether the trend is the same for each of the visuospatial tasks. For mental rotation the difference between males and females was significant, $F(1,313)=18.15, p<.001, \eta_{p}^{2}=.06$. However, differences in localization and form completion tasks were not statistically significant, $F(1,313)=2.44, p>.05, \eta_{\mathrm{p}}{ }^{2}=.01$, for localization, and $F(1,313)=0.44$, $p>.05, \eta_{\mathrm{p}}^{2}=.00$, for form completion. There was no multivariate effect of age group on the three dependent variables, Wilks' lambda $=1.00$, $F(3,311)=0.02, p>.05, \eta_{\mathrm{p}}{ }^{2}=.00$. Nevertheless, the interaction between sex and age group was statistically significant, Wilks' lambda $=.97, F(3$, $311)=2.97, p<.05, \eta_{p}^{2}=.03$. Each dependent variable was subjected to a further ANOVA in order to examine the interaction between sex and age group on each visuospatial task. The interaction between sex and age group was nonsignificant for localization, $F(1,317)=0.09, p>.05$, $\eta_{\mathrm{p}}{ }^{2}=.00$, and for form completion, $F(1,317)=0.44, p>.05, \eta_{\mathrm{p}}{ }^{2}=.00$. However, there was a statistically significant interaction between sex and age group on mental rotation, $F(1,317)=8.78, p<.01, \eta_{\mathrm{p}}{ }^{2}=.03$; see Figure 3. In mental rotation, simple main effects analysis showed that adult males outperformed adult females $(p<.001)$, whereas there were no sex differences in children $(p>.05)$.

\section{TABLE 2.}

Means (SD, z-Scores, Raw Scores) and Cohen's $d$ For Sex Differences in Verbal and Visuospatial Cognitive Abilities

\begin{tabular}{|c|c|c|c|c|c|c|}
\hline & \multicolumn{3}{|c|}{ Children } & \multicolumn{3}{|c|}{ Adults } \\
\hline & Boys $(N=82)$ & Girls $(N=87)$ & $d$ & $\operatorname{Men}(N=77)$ & Women $(N=80)$ & $d$ \\
\hline \multicolumn{7}{|l|}{ Verbal ability } \\
\hline \multicolumn{7}{|l|}{ Serial sounds } \\
\hline$z$-scores & $-0.13(0.92)$ & $0.12(1.06)$ & 0.25 & $-0.04(1.13)$ & $0.04(0.86)$ & 0.08 \\
\hline raw scores & $81.96(37.66)$ & $91.98(43.07)$ & & $111.09(55.34)$ & $114.68(42.21)$ & \\
\hline \multicolumn{7}{|l|}{ Serial digits } \\
\hline$z$-scores & $-0.26(1.01)$ & $0.07(1.00)$ & 0.32 & $-0.11(0.59)$ & $0.06(0.85)$ & 0.23 \\
\hline raw scores & $7.72(2.57)$ & $7.95(2.56)$ & & $9.58(1.06)$ & $9.88(1.58)$ & \\
\hline \multicolumn{7}{|l|}{ Verbal fluency } \\
\hline$z$-scores & $-0.31(0.94)$ & $0.29(0.97)$ & $0.63^{* * *}$ & $-0.25(0.97)$ & $0.24(0.98)$ & $0.50^{\star *}$ \\
\hline raw scores & $17.80(6.47)$ & $21.93(6.65)$ & & $39.43(10.69)$ & $44.88(10.82)$ & \\
\hline \multicolumn{7}{|l|}{ Visuospatial ability } \\
\hline \multicolumn{7}{|l|}{ Mental rotation } \\
\hline$z$-scores & $0.07(0.99)$ & $-0.07(1.01)$ & 0.14 & $0.39(0.94)$ & $-0.39(0.92)$ & $0.84^{* * *}$ \\
\hline raw scores & $15.41(5.74)$ & $14.98(5.54)$ & & $15.23(4.10)$ & $11.35(4.57)$ & \\
\hline \multicolumn{7}{|l|}{ Localization } \\
\hline$z$-scores & $-0.10(0.88)$ & $0.11(1.08)$ & 0.21 & $-0.06(1.01)$ & $0.08(1.00)$ & 0.14 \\
\hline raw scores & $1.93(0.36)$ & $2.03(0.45)$ & & $0.98(0.74)$ & $1.06(0.72)$ & \\
\hline \multicolumn{7}{|l|}{ Form completion } \\
\hline$z$-scores & $0.04(0.97)$ & $0.04(1.01)$ & 0.00 & $0.10(1.01)$ & $-0.05(0.99)$ & 0.15 \\
\hline raw scores & $13.43(7.15)$ & $13.67(7.17)$ & & $12.18(4.38)$ & $11.34(4.33)$ & \\
\hline
\end{tabular}

Note. The localization score is higher for poorer performance; all cognitive ability tasks were standardized within age groups. ${ }^{* *} p<.01{ }^{* * *} p<.001$ 


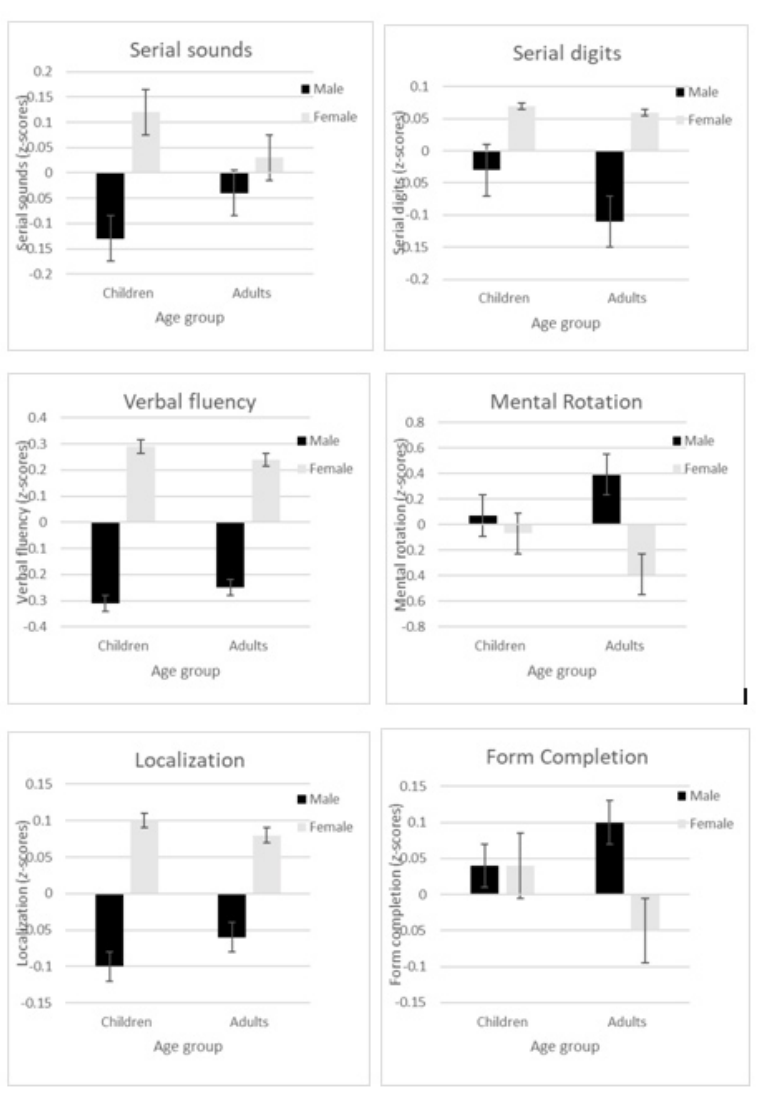

FIGURE 4.

Means $( \pm S E$ ) of $z$-scores for verbal cognitive tasks: serial sounds, serial digits, and verbal fluency; for visuospatial cognitive tasks: mental rotation, localization, and form completion.

\section{DISCUSSION}

The present study aimed at investigating developmental patterns in sex differences on cognitive abilities. Specifically, the present study assessed sex differences in visuospatial and verbal abilities in children and adults. The hypotheses were partially supported. Sex differences in verbal fluency were identified in childhood as well as in adulthood, with females outperforming males. This finding is in accord with previous results demonstrating the emergence of sex differences in verbal abilities in childhood, with no indication of these differences magnified throughout development. For example, in a cross-sectional study of children aged 12-14, Herlitz et al. (2013) documented sex differences in verbal fluency without any indication for a larger magnitude during adolescence. The authors suggested that, in contrast to the held notion that sex differences are magnified during adolescence, supported by evidence related to sex differences in cortical development (Lenroot \& Giedd, 2010), their findings, joined with the present findings, indicate that sex differences in verbal abilities emerge before puberty and imply that the source of these differences should be sought early in development. Findings on other verbal tasks (short-term memory tasks) failed to produce significant results. This is line with the empirical evidence with regard to sex differences in memory tasks in children, usually implying no sex differences (e.g., Alloway et al., 2006; Palejwala \& Goldenring Fine, 2015). In contrast, our findings do not support previous findings of sex differences in memory among adult participants. Previous studies have demonstrated female superiority in various memory tasks, such as short-term word-recognition-memory (Weiss et al., 2003), verbal learning and short-term memory (Bleecker et al., 1988), and free recall (Kramer et al., 1988). Nevertheless, other studies failed to document the female superiority; for example, in a serial digits task and in a digit backward task, men and women did not differ significantly (Duff \& Hampson, 2001). This finding may be due to the composition of our adult sample. Although the sample consisted of participants from various departments, participants from disciplines, such as Mathematics and Natural Sciences, were not represented in the present study. Vocational interest was linked to dimensions related to people versus things orientations (Lippa, 1998). Among the underlying mechanism for these orientations are cognitive abilities. It has been suggested, for example, that memory abilities are associated with the people orientation that, in turn, is influential in vocational interest related to social studies (Halpern et al., 2007). Perhaps the limited representation of participants from Science, Technology, Engineering, and Mathematics (STEM) fields may account for the lack of sex differences in memory in the present study.

With regard to visuospatial tasks, empirical literature reported consistently that the largest effect size of sex differences was found for the mental rotation task (Levine, Foley, Lourenco, Ehrlich, \& Ratliff, 2016). The present study aimed at capturing the developmental pattern of the emergence of sex differences in mental rotation. The findings revealed that sex differences are not apparent in children. In adults, our findings supported previous studies demonstrating a large effect size $(d=0.84)$. The present study's task was based on Shepard and Metzler's (1971) three-dimensional mental rotation task, in which participants are required to mentally rotate an object in three dimensions to determine its matching to one of several other objects. Previous meta-analyses (Linn \& Peterson, 1985; Voyer et al., 1995) confirmed that there is substantial male advantage on mental rotation tasks and pointed out that the tasks which involve 3D rotations (as opposed to rotating simpler shapes in the picture plane) produce the largest effect size, probably due to females' difficulty to mentally rotate objects in depth. In children, the present study used 2D stimuli, which have not produced significant sex differences. Ruthsatz, Neuburger, Jansen, and Quaiser-Pohl (2014) found that 10-year-old boys outperformed girls in tasks with 3D cube figures rotated in-depth. Nevertheless, other studies suggested that 3D tasks may be too cognitively demanding for children (Hoyek et al., 2012; Jansen et al., 2013). For example, studies that included 3D cubes tasks alongside 2D tasks showed that children encountered difficulties performing the 3D cubes task and concluded that this task is inappropriate for elementary school children (e.g., Jansen et al., 2013). Therefore, a line of studies used various versions of $2 \mathrm{D}$ tasks, which are generally found to be easier and faster than 3D tasks (Hoyek et al., 2012). Still, the need for established paradigms appropriate for elementary school children was apparent. 
Several studies tested children's performance on various 2D stimuli using animal drawings (Kucian et al., 2007) or other familiar objects (Ruthsatz, Neuburger, Jansen, \& Quaiser-Pohl, 2015), alphanumeric stimuli (Hoyek et al., 2012), or abstract characters (Hoyek et al., 2012; Kail, Pellegrino, \& Carter, 1980). They have demonstrated that various 2D stimuli differ in their difficulty level. Performances for familiar 2D stimuli (e.g., animal drawings or alphanumeric stimuli) were found to elicit higher accuracy scores and shorter response times as opposed to abstract characters (e.g., Kail et al., 1980). Kail et al. (1980) proposed that abstract characters are unfamiliar to both children and adults as opposed to familiar (e.g., alphanumeric) stimuli. Encoding of a familiar stimulus involves activation of an internal labelable pattern, whereas encoding of an unfamiliar stimulus, which does not correspond to an internal standard, requires much more processing resources, resulting in poorer performance in accuracy and response time. Moreover, Hoyek et al. (2012) showed that middle school children outperformed elementary school children in both 3D cubes and 2D abstract characters, providing support to the $2 \mathrm{D}$ abstract stimuli as valid stimuli assessing mental rotation performance in children. Therefore, in the present study, in order to use age-appropriate and -comparable paradigms, a 3D cubes task was used in adults and a $2 \mathrm{D}$ abstract characters task (which has been proven at the highest difficulty level among 2D stimuli) was used in children. Previous studies in school-aged children using various $2 \mathrm{D}$ stimuli provided mixed results for sex differences in mental rotation (e.g., Neuburger et al., 2011; Titze, Jansen, \& Heil, 2010). However, studies using $2 \mathrm{D}$ abstract characters did not find sex differences in this task. For example, Hoyek et al. (2012) showed that boys outperformed girls in abstract $2 \mathrm{D}$ stimuli and in $3 \mathrm{D}$, but only in the middle school group, and not in the elementary school group. The authors even suggested that these stimuli involving abstract characters are difficult to encode and mentally rotate, and therefore might be too complex for elementary school children. Collins and Kimura (1997) provided further support to the notion that the $2 \mathrm{D}$ tasks are easier than 3D tasks. They found that men outperformed women both on the $3 \mathrm{D}$ cubes task and on the abstract 2D task, and that performance on these tasks was significantly correlated. Thus, contradictory premises arise of the complexity of abstract $2 \mathrm{D}$ stimuli. Therefore, in the current study, a further analysis was conducted examining the variance differences between the two age groups in mental rotation performance. A significant difference was found $(F=4.43, p=.036)$, with higher variability in the performance of children in comparison with that of adults. That is, premises regarding the complexity degree of the $2 \mathrm{D}$ task were ruled out in the present sample. Children exhibited variance in their performance, and it was not due to sex differences. The present findings support former studies using abstract stimuli in the notion that sex differences in mental rotation abilities are magnified throughout development. In their meta-analysis, Voyer et al. (1995) showed an increase in effect size (Cohen's $d$ ) from 0.33 (in children aged less than 13) to 0.45 (in adolescents aged 13-18) and 0.66 (in adults). Our results supported this line of findings with a somewhat smaller effect size in children (0.14) and a larger effect size in adults (0.84).
The underlying mechanism for the developmental pattern of sex differences' emergence in mental rotation received much attention from a biological perspective, especially the role of sex hormones and sex differences in brain structure and function. The most notable influence of sex hormones occurs during sensitive periods in development: prenatal and postnatal (Collaer et al., 2007; Halpern, 2012). Puberty has been suggested as a sensitive period of sex hormone-dependent brain organization, with increased levels of sex hormones secretion influencing changes in cognitive performance (Berenbaum \& Beltz, 2011; Shangguan \& Shi, 2009). Another source of support for the assumption regarding magnified sex differences in mental rotation arises from studies on sex differences in brain structure and function. For example, in studies focusing on brain structure, sex differences in the parietal lobe-a brain region involved in spatial ability-have been demonstrated (e.g., Culham \& Kanwisher, 2001). Furthermore, the activation patterns during spatial tasks seem to be sexually dimorphic as well (e.g., Butler et al., 2006). One developmental fMRI study addressed developmental patterns in brain activation (Kucian et al., 2007). No significant sex differences in mental rotation performance in adults or in children were found. Nevertheless, effect sizes were largely different with 0.21 for third-grade children and 0.90 for adults. Furthermore, while no sex differences in brain activation were found in children, sex differences in brain activation were demonstrated in adults. These brain regions contain significant populations of sex hormones (Bramen et al., 2011). In a recent review, Levine et al. (2016) have postulated an integrative approach, including biological as well as environmental factors accounting for sex differences in mental rotation abilities. The suggested model could explain the differences in magnitude from childhood through adulthood in sex differences in mental rotation shown in the present study, as well as in former studies. One possible integrative attempt regarding Gene $\times$ Environment interaction influencing mental rotation performance suggests that the preliminary male advantage in these tasks led them to become more interested in these activities, which in turn led to a greater spatial advantage (Pezaris \& Casey, 1991). This notion is also supported by an evolutionary approach suggesting that differing evolutionary forces imposed on human males and females resulted in differences in anatomy followed by sexual differences in function (Lenroot \& Giedd, 2010). Males engaged in more spatially demanding tasks and, therefore, differential corresponding neuroendocrine infrastructures were needed to develop in human males and females.

To summarize, the present findings suggest that whereas females outperform males in verbal fluency throughout development, sex differences favoring males occur after puberty. Although the findings regarding verbal fluency are not consistent across studies, studies concerning the emergence and increase of sex differences in spatial abilities, and especially mental rotation ability, seem consistent (Berenbaum \& Beltz, 2011). Four decades ago, Waber (1976) postulated that sex differences in cognitive abilities could be explained by maturation rate. He suggested that through the mediation role of the development of hemispheric specialization, late maturers have better spatial abilities, 
whereas early maturers have better verbal abilities. The present findings provide support for this suggestion.

The present study has some limitations. First, the nature of the mental rotation task used for children and adults in the current study was different. Although, as elaborated earlier, the abstract 2D measure for mental rotation capability in children has been validated as capturing a high level of complexity of mental rotation ability, still, future studies are needed for developing 3D stimuli appropriate for children. Lately, there has been an attempt to develop a 3D mental rotation measure appropriate for young children. Hawes, LeFevre, $\mathrm{Xu}$, and Bruce (2015) designed tangible 3D wooden block figures as stimuli based on the 3D cube figures by Shepard and Metzler (1971). Using this task with 2D stimuli, the authors did not document sex differences in children aged 4-8 years. Future studies should follow in this vein and continue uncovering the cognitive mechanisms required for performance in $3 \mathrm{D}$ mental rotation tasks, thus leading to the development of age-appropriate 3D stimuli. Second, we have not controlled for the time of day or stage in the menstrual cycle of data collection. Since testosterone, estrogen, and progesterone vary across day time and the menstrual cycle, further studies should control for these factors. Third, although the adult sample consisted of participants from various departments (including social as well as formal sciences), future studies should still examine a wide range of study disciplines, especially from natural sciences and STEM fields in order to uncover the influence of field orientation and field expertise on the magnitude of sex differences in various cognitive abilities. Quaiser-Pohl and Lehmann (2002) found male superiority in mental rotation tasks, but the effect sizes varied across academic programs, with highest effect size in humanities and social sciences and lowest in computational visualization students. The authors suggested that their findings have considerable consequences for intervention programs bridging the sex gap in spatial abilities. Given the present findings suggesting sex differences also in verbal abilities, it is important to broaden the sampling of participants from various fields and examine other cognitive abilities in order to evaluate the potential role of interventions in a variety of cognitive abilities.

The present findings suggesting a differential course of development for differing cognitive abilities call for elaboration in deepening the search for the development pattern of sex differences in cognitive abilities. Several studies addressed this question using cross-sectional samples in childhood and in early puberty (e.g., Herlitz et al., 2013). However, in order to uncover the exact pattern of the development of sex differences in cognitive abilities, a longitudinal study is still needed. Furthermore, the socioeconomic status of children has not been found to relate to the present findings. Previous studies have demonstrated that cognitive skills may vary as a function of socioeconomic function. For example, Levine, Vasilyeva, Lourenco, Newcombe, and Huttenlocher (2005) have demonstrated that in middle-high socioeconomic groups, there were sex differences in spatial abilities, whereas no differences were found in low socioeconomic groups. It has been suggested that higher socioeconomic status boys have much more access to spatial activities in comparison with lower socioeconomic boys. This suggestion implies that there is an important role for exposure and training in cognitive abilities improvement (Levine et al., 2016). Nevertheless, since the present findings did not support this environmental influence, and in light of the controversies in the empirical literature, future studies should control these variables..

\section{REFERENCES}

Alloway, T. P., Gathercole, S. E., \& Pickering, S. J. (2006). Verbal and visuospatial short-term and working memory in children. Child Development, 77, 1698-1716. doi: 10.1111/j.1467-8624 .2006.00968.x $\overline{\mathrm{WWW}}$

Barel, E., \& Tzischinsky, O. (2017). The role of sex hormones and of 2D:4D ratio in individual differences in cognitive abilities. Journal of Cognitive Psychology, 29, 497-507. doi: 10.1080/20445911.2017.1279166

Berenbaum, S. A., \& Beltz, A. M. (2011). Sexual differentiation of human behavior: Effects of prenatal organizational hormones. Frontiers in Neuroendocrinology, 32:10. doi: 10.1016/j. yfrne.2011.03.001 WWW

Bleecker, M. L., Bolla-Wilson, K., Agnew, J., \& Meyers, D. A. (1988). Age-related sex differences in verbal memory. Journal of Clinical Psychology, 44, 403-411. doi: 10.1002/1097-4679-

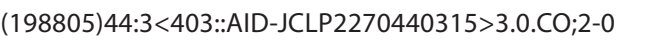

Bramen, J. E., Hranilovich, J. A., Dahl, R. E., Forbes, E. E., Chen, J., Toga, A. W., \& Sowell, E. R. (2011). Puberty influences medial temporal lobe and cortical gray matter maturation differently in boys than girls matched for sexual maturity. Cerebral Cortex, 21, 636-646. doi: 10.1093/cercor/bhq137 WWW

Burton, L. A., \& Henninger, D. (2013). Sex differences in relationships between verbal fluency and personality. Current Psychology, 32, 168-174. doi: 10.1007/s12144-013-9167-4

Butler, T., Imperato-McGinley, J., Pan, H., Voyer, D., Cordero, J., Zhu, Y-S., Stern, E., \& Silbersweig, D. (2006). Sex differences in mental rotation:Top-down versus bottom-up processing. Neurolmage, 32, 445-456. doi: 10.1016/j.neuroimage.2006.03.030 |wWW

Caldera, Y. M., Huston, A. C., \& O'Brien, M. (1989). Social interactions and play patterns of parents and toddlers with feminine, masculine, and neutral toys. Child Development, 60, 70-76. doi: $10.2307 / 1131072 \underline{\underline{W W}}$

Caldera, Y. M., Mc Culp, A., O'Brien, M., Truglio, R. T., Alvarez, M., \& Huston, A. C. (1999). Children's play preferences, construction play with blocks, and visual-spatial skills: Are they related? International Journal of Behavioral Development, 23, 855-872. doi: 10.1080/016502599383577

Carlson, L. E., \& Sherwin, B. B. (2000). Higher levels of plasma estradiol and testosterone in healthy elderly man compared with aged-matched women may protect aspects of explicit memory. Menopause, 7, 80-88. doi: 10.1080/13685530008500332

Cherrier, M. M., Asthana, S., Plymate, S., Baker, L., Matsamoto, A., Peskind, E., \& LaTendresse, S. (2001). Testosterone supplementation improve spatial and verbal memory in healthy older men. Neurology, 57, 80-88. doi: 10.1212/WNL.57.1.80 
Collaer, M. L., Reimers, S., \& Manning, J. T. (2007). Visuospatial performance on an internet line judgment task and potential hormonal markers: Sex, sexual orientation, and 2d:4d. Archives of Sexual Behavior, 36, 177-192. doi: 10.1007/s10508-0069152-1

Collins, D. W., \& Kimura, D. (1997). A large sex difference on a twodimensional mental rotation task. Behavioral Neuroscience, 111, 845-849. doi: 10.1037/0735-7044.111.4.845 [WWW

Culham, J. C., \& Kanwisher, N. G. (2001). Neuroimaging of cognitive functions in human parietal cortex. Current Opinion in Neurobiology, 11, 157-163. doi: 10.1016/S0959-

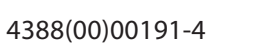

Driscoll, I., Hamilton, D. A., Yeo, R. A., Brooks, W. M., \& Sutherland, R. J. (2005). Virtual navigation in humans: The impact of age, sex, and hormones on place learning. Hormones and Behavior, 47, 326-35. doi: 10.1016/j.yhbeh.2004.11.013 wWw

Duff, S. J., \& Hampson, E. (2001). A sex difference on a novel spatial working memory task in humans. Brain and Cognition, 47, 470-493. doi: 10.1006/brcg.2001.1326 WWW

Frick, A., Ferrara, K., \& Newcombe, N. S. (2013). Using a touch screen paradigm to assess the development of mental rotation between 3.5 and 5.5 years of age. Cognitive Processing, 14, 117-127. doi: 10.1007/s10339-012-0534-0 WwW

Geary, D. C. (2010). Male, female: The evolution of human sex differences (2nd ed.). Washington, D. C.: American Psychological Association.

Gordon, H. W. (1986). The cognitive laterality battery: Tests of specialized cognitive function. International Journal of Neuroscience, 29, 223-244. doi: 10.3109/00207458608986153 WWW

Gordon, H. W., \& Harness, B. Z. (1977). A test battery for diagnosis and treatment of developmental dyslexia. DASH Speech and Hearing Disorders, 8, 1-7.

Gordon, H. W., \& Lee, P. A. (1993). No difference in cognitive performance between phases of the menstrual cycle. Psychoneuroendocrinology, 18, 521-531. doi: 10.1016/03064530(93)90045-M WwW

Halari, R., Hines, M., Kumari, V., Mehrotra, R., Wheeler, M., Ng, V., \& Sharma, T. (2005). Sex differences and individual differences in cognitive performance and their relationship to endogenous gonadal hormones and gonadotropins. Behavioral Neuroscience, 119, 104-117. doi: 10.1037/0735-7044.119.1.104

Halpern, D. F. (2012). Sex differences in cognitive abilities (4th ed.). New York, NY: Psychology Press.

Halpern, D. F., Benbow, C. P., Geary, D. C., Gur, R. C., Shilbey Hyde, J., \& Gernsbacher, M. A. (2007). The science of sex differences in science and mathematics. Psychological Science in the Public Interest, 8, 1-51. doi: 10.1111/j.1529-1006.2007.00032.x WwW

Hausmann, M., Slabbekoorn, D., Van Goozen, S. H. M., CohenKettenis, P. T., \& Güntürkün, O. (2000). Sex hormones affect spatial abilities during the menstrual cycle. Behavioral Neuroscience, 114, 1245-1250. doi: 10.1037/0735-7044.114.6.1245 Www
Hawes, Z., LeFevre, J., Xu, C., \& Bruce, C. D. (2015). Mental rotation with tangible three-dimensional objects: A new measure sensitive to developmental differences in 4- to 8-year-old children. Mind, Brain, \& Education, 9, 10-18. doi: 10.1111/mbe.12051

Herlitz, A., Reuterskiöld, L., Lovén, J., Thilers, P. P., \& Rehnman, J. (2013). Cognitive sex differences are not magnified as a function of age, sex hormones, or puberty development during early adolescence. Developmental Neuropsychology, 38, 167-179. doi: 10.1080/87565641.2012.759580 WWW

Hespos, S. J., \& Rochat, P. (1997). Dynamic mental representation in infancy. Cognition, 64, 153-188. doi: 10.1016/S00100277(97)00029-2 www

Hines, M. (2004). Brain gender. Oxford, England: Oxford University Press.

Hines, M. (2011). Gender development and the human brain. Annual Review of Neuroscience, 34, 69-88. doi: 10.1146/annurev-neuro-061010-113654

Hines, M., Fane, B. A., Pasterski, V. L., Mathews, G. A., Conway, G. S., \& Brook, C. (2003). Spatial abilities following prenatal androgen abnormality: Targeting and mental rotations performance in individuals with congenital adrenal hyperplasia. Psychoneuroendocrinology, 28, 1010-1026. doi: 10.1016/ S0306-4530(02)00121-X WWW

Hoyek, N., Collet, C., Fargier, P., \& Guillot, A. (2012). The use of the Vandenberg and Kuse Mental Rotation Test in Children. Journal of Individual Differences, 33, 62-67. doi: 10.1027/16140001/a000063

Hyde, J. S., \& Linn, M. C. (1988). Gender differences in verbal abilities: A meta-analysis. Psychological Bulletin, 104, 53-69. doi: 10.1037/0033-2909.104.1.53

lachini, T., Sergi, I., Ruggiero, G., \& Gnisci, A. (2005). Gender differences in object location memory in a real three-dimensional environment. Brain and Cognition, 59, 52-59. doi: 10.1016/j. bandc.2005.04.004 WwW

Jansen, P., Schmelter, A., Quaiser-Pohl, C., Neuburger, S., \& Heil, M. (2013). Mental rotation performance in primary school age children: Are there gender differences in chronometric tests? Cognitive Development, 28, 51-62. doi: 10.1016/j. cogdev.2012.08.005

Kail, R., Pellegrino, J., \& Carter, P. (1980). Developmental changes in mental rotation. Journal of Experimental Child Psychology, 29, 102-116. doi: 10.1016/0022-0965(80)90094-6

Keith, T. Z., Reynolds, M. R., Roberts, L. G., Winter, A. L., \& Austin, C. A. (2011). Sex differences in latent cognitive abilities ages 5 to 17: Evidence from the Differential Ability Scales (2nd ed). Intelligence, 39, 389-404. doi: 10.1016/j.intell.2011.06.008

Kimura, D. (2002). Sex hormones influence human cognitive pattern. Neuroendocrinology Letters, 23, 68-77.

Kramer, J. H., Delis, D. C., \& Daniel, M. (1988). Sex differences in verbal learning. Journal of Clinical Psychology, 44, 907-915. doi: 10.1002/1097-4679(198811)44:6<907::AIDJCLP2270440610>3.0.CO;2-8 
Kucian, K., von Aster, M., Loenneker, T., Dietrich, T., Mast, F. W., \& Martin, E. (2007). Brain activation during mental rotation in school children and adults. Journal of Neural Transmission, 114, 675-686. doi: 10.1007/s00702-006-0604-5 WWW

Lenroot, R. K., \& Giedd, J. N. (2010). Sex differences in the adolescent brain. Brain and Cognition, 72, 46-55. doi: 10.1016/j. bandc.2009.10.008 WWW

Lenroot, R. K., Gogtay, N., Greenstein, D. K., Wells, E. M., Wallace, G. L., Clasen, L. S., . . Giedd, J. N. (2007). Sexual dimorphism of brain developmental trajectories during childhood and adolescence. Neurolmage, 36, 1065-1073. doi: 10.1016/j. neuroimage.2007.03.053 WWW

Levine, S. C., Foley, A., Lourenco, S., Ehrlich, S., \& Ratliff, K. (2016). Sex differences in spatial cognition: Advancing the conversation. WIREs Cognitive Science, 7, 127-155. doi: 10.1002/ WCS. $1380 \underline{\underline{W W}}$

Levine, S. C., Vasilyeva, M., Lourenco, S. F., Newcombe, N. S., \& Huttenlocher, J. (2005). Socioeconomic status modifies the sex difference in spatial skill. Psychological Science, 16, 841-845. doi: 10.1111/j.1467-9280.2005.01623.x WWW

Linn, M. C., \& Petersen, A. C. (1985). Emergence and characterization of sex differences in spatial ability: A meta-analysis. Child Development, 56, 1479-1498. doi: 10.2307/1130467 WW

Lippa, R. (1998). Gender-related individual differences and the structure of vocational interests of the people-things dimension. Journal of Personality and Social Psychology, 74, 996-1009. doi: 10.1037/0022-3514.74.4.996 WWW

Lutchmaya, S., Baron-Cohen, S., \& Raggatt, P. (2002). Foetal testosterone and vocabulary size in 18 and 24 month old infants. Infant Behavior and Development, 24, 418-424. doi: 10.1016/ S0163-6383(02)00087-5

Möhring, W., \& Frick, A. (2013). Touching up mental rotation: Effects of manual experience on 6-month-old infants' mental object rotation. Child Development, 84, 1554-1565. doi: 10.1111/cdev.12065 WWW

Moore, D. S., \& Johnson, S. P. (2008). Mental rotation in human infants: A sex difference. Psychological Science, 19, 1063-1066. doi: 10.1111/j.1467-9280.2008.02200.x WWW

Neuburger, S., Jansen, P., Heil, M., \& Quaiser-Pohl, C. (2011). Gender differences in pre-adolescents' mental-rotation performance: Do they depend on grade and stimulus type? Personality and Individual Differences, 50, 1238-1242. doi: 10.1016/j.paid.2011.02.017

Palejwala, M. H., \& Goldenring Fine, J. (2015). Gender differences in latent cognitive abilities in children aged 2 to 7 . Intelligence, 48, 96-108. doi: 10.1016/j.intell.2014.11.004

Parsons, T. D., Larson, P., Kratz, K., Thiebaux, M., Bluestein, B., Buckwalter, J. G., \& Rizzo, A. A. (2004). Sex differences in mental rotation and spatial rotation in a virtual environment. Neuropsychologia, 42, 555-562. doi: 10.1016/j.

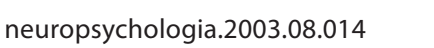

Peters, M., Manning, J., \& Reimers, S. (2007). The effects of sex, sexual orientation, and digit ratio (2D:4D) on mental rotation performance. Archives of Sexual Behavior, 36, 251-260. doi:

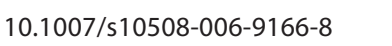

Pezaris, E., \& Casey, B. M. (1991). Girls who use "masculine" problem-solving strategies on a spatial task: Proposed genetic and environmental factors. Brain and Cognition, 17, 1-22. doi: 10.1016/0278-2626(91)90062-D WWW

Quaiser-Pohl, C., \& Lehmann, W. (2002). Girls' spatial abilities: Charting the contributions of experiences and attitudes in different academic groups. British Journal of Educational

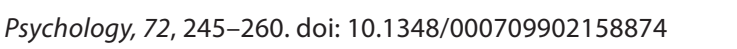

Quinn, P. C., \& Liben, L. S. (2008). A sex difference in mental rotation in young infants. Psychological Science, 19, 1067-1070.

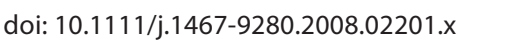

Ruthsatz, V., Neuburger, S., Jansen, P., \& Quaiser-Pohl, C. (2014). Pellet figures, the feminine answer to cube figures? Influence of stimulus features and rotational axis on the mental-rotation performance of fourth-grade boys and girls. In: C. Freksa, B. Nebel, M. Hegarty, \& T. Barkowsky (Eds.), Spatial cognition IX. Spatial cognition 2014. Lecture notes in computer science (Vol. 8684, pp. 372-380). Cham, Switzerland: Springer. doi: 10.1007/978-3-319-11215-2_26

Ruthsatz, V., Neuburger, S., Jansen, P., \& Quaiser-Pohl, C. (2015). Cars or dolls? Influence of the stereotyped nature of the items on children's mental-rotation performance. Learning and Individual Differences, 43, 75-82. doi: 10.1016/j. lindif.2015.08.016

Shangguan, F. F., \& Shi, J. N. (2009). Puberty timing and fluid intelligence: A study of correlations between testosterone and intelligence in 8- to 12-year-old Chinese boys. Psychoneuroendocrinology, 34, 983-988. doi: 10.1016/j. psyneuen.2009.01.012 $\overline{\mathrm{WWW}}$

Shepard, R. N., \& Metzler, J. (1971, February). Mental rotation of three-dimensional objects. Science, 171(3972), 701-703. doi: 10.1126/science.171.3972.701 WwW

Titze, C., Jansen, P., \& Heil, M. (2010). Mental rotation performance and the effect of gender in fourth graders and adults. European Journal of Developmental Psychology, 7, 432-444. doi: 10.1080/17405620802548214

Voyer, D., Voyer, S., \& Bryden, M. P. (1995). Magnitude of sex differences in spatial abilities: A meta-analysis and consideration of critical variables. Psychological Bulletin, 117, 250-270. doi: 10.1037/0033-2909.117.2.250 $\underline{\underline{\omega W}}$

Waber, D. P. (1976, May). Sex differences in cognition: A function of maturation rate? Science, 192(4239), 572-574. doi: 10.1126/ science.1257795 $\overline{\mathrm{WWW}}$

Weiss, E. M., Kemmler, G., Deisenhammer, E. A., Fleischhacker, W. W., \& Delazer, M. (2003). Sex differences in cognitive functions. Personality and Individual Differences, 35, 863-875. doi: 10.1016/S0191-8869(02)00288-X 
Zambrana, I. M., Ystrom, E., \& Pons, F. (2012). Impact of gender, maternal education, and birth order on the development of language comprehension: A longitudinal study from 18 to 36 months of age. Journal of Developmental and Behavioral Pediatrics, 33, 146-155. doi: 10.1097/DBP.0b013e31823d4f83 |Www

RECEIVED 04.09.2017 | ACCEPTED 19.02.2018 